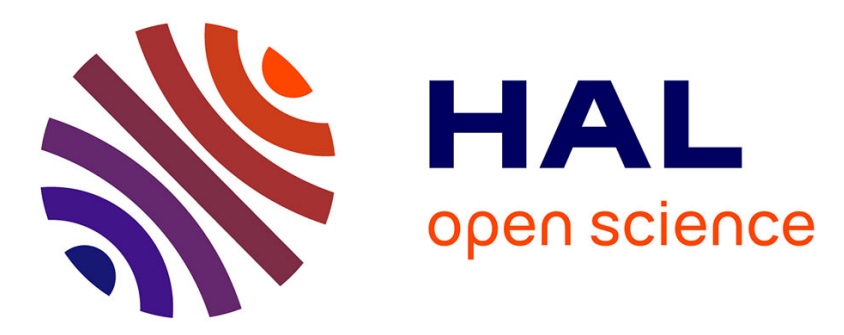

\title{
Soft-sell versus hard-sell: quelle approche publicitaire dans les pays arabo-musulmans? Une étude exploratoire au Maroc et en Arabie Saoudite
} Habiba Elbardai

\section{- To cite this version:}

Habiba Elbardai. Soft-sell versus hard-sell: quelle approche publicitaire dans les pays arabomusulmans? Une étude exploratoire au Maroc et en Arabie Saoudite. Projectics / Proyéctica / Projectique, 2020, 3 (27), pp.75-96. 10.3917/proj.027.0075 . hal-03020489

\section{HAL Id: hal-03020489 \\ https://hal.science/hal-03020489}

Submitted on 23 Nov 2020

HAL is a multi-disciplinary open access archive for the deposit and dissemination of scientific research documents, whether they are published or not. The documents may come from teaching and research institutions in France or abroad, or from public or private research centers.
L'archive ouverte pluridisciplinaire HAL, est destinée au dépôt et à la diffusion de documents scientifiques de niveau recherche, publiés ou non, émanant des établissements d'enseignement et de recherche français ou étrangers, des laboratoires publics ou privés. 


\title{
Soft-sell versus hard-sell : quelle approche publicitaire dans les pays arabo- musulmans ? Une étude exploratoire au Maroc et en Arabie Saoudite.
}

\author{
ELBARDAI Habiba
}

Doctorante à l'Université de Lorraine, Laboratoire CEREFIGE, France

Chargée de cours à l'Université Internationale d'Agadir, Universiapolis-Maroc

E-mail : h.elbardai@,centaure-tudor.comTél. : 00212666018423

\section{Résumé :}

Le but de cet article est de mettre en évidence les pratiques publicitaires dans le contexte arabomusulman. Nous présenterons les tendances les plus apparentes des annonceurs en faisant référence aux approches publicitaires soft-sell et hard-sell. Une analyse de contenu de publicités au Maroc et en Arabie saoudite s'est effectuée en suivant une grille d'observation fondée sur la théorie d'Okazaki et al. (2010). Les résultats affichent que les pratiques publicitaires au Maroc et en Arabie-saoudites sont assez similaires et tendent vers la présentation des éléments factuels de l'offre. La conclusion montre les implications managériales et les pistes de recherche qui ont émergé de cette étude.

\section{Mots clé :}

Message soft-sell, message hard-sell, communication persuasive, arabo-musulman. 


\title{
Soft-sell versus hard-sell : quelle approche publicitaire dans les pays arabo- musulmans? Une étude exploratoire au Maroc et en Arabie Saoudite.
}

\section{Résumé :}

Cet article vise à étudier les pratiques publicitaires dans le contexte arabo-musulman, et plus particulièrement, à identifier si une approche publicitaire (soft-sell versus hard-sell) est privilégiée par les annonceurs. En s'appuyant sur une grille d'observation construite à l'aide de la théorie d'Okazaki et al. (2010), une analyse de contenu est menée sur un corpus de 18 publicités diffusées au Maroc et en Arabie Saoudite. Les résultats montrent que les pratiques publicitaires au Maroc et en Arabie Saoudite sont relativement similaires et s'orientent vers l'adoption d'une approche publicitaire hard-sell qui se focalise essentiellement sur la présentation des éléments factuels de l'offre. En conclusion, sont soulignés les apports de cette recherche, ses limites, ses implications managériales ainsi que les voies de recherche qui émergent de cette étude exploratoire.

\section{Mots clé :}

Approche publicitaire soft-sell, Approche publicitaire hard-sell, communication persuasive, contexte arabo-musulman

\begin{abstract}
This article's main objective is to study advertising practices in the Arab-Muslim context, and more specifically, to identify whether an advertising approach (soft-sell versus hard-sell) is preferred by advertisers. Based on an observation grid constructed using the theory of Okazaki al. (2010), a content analysis is carried out on a corpus of 18 advertisements broadcasted in Morocco and Saudi Arabia. The results show that advertising practices in Morocco and Saudi Arabia are relatively similar and are moving towards the adoption of a hard-sell advertising approach which essentially focuses on the presentation of the factual elements of the offer. In conclusion, the contributions of this research, its limits, its managerial implications and the lines of research that emerge from this exploratory study are highlighted.
\end{abstract}

\section{Keywords:}

soft-sell appeal, hard-sell appeal, persuasive communication, Arabo-Muslim context. 


\section{Introduction}

Le développement économique de l'entreprise et la conquête des marchés internationaux soulèvent subséquemment la question du discours publicitaire à adopter, oscillant entre la standardisation à l'image du « what else ? » qui constitue un facteur déterminant du succès de la marque Nespresso à travers le monde et l'adaptation à la culture locale à l'instar de la stratégie publicitaire retenue par les marques Coca-Cola ou Mc Donalds' (Butt, Mukerji et Shareef, 2017 ; Zhou, Poon et Wang, 2015). Que doivent dire les marques ? Et comment doivent-elles le dire?

La littérature en communication marketing introduit une dichotomie dans l'approche publicitaire en distinguant une approche publicitaire soft-sell fondée sur un discours émotionnel et une approche publicitaire hard-sell s'appuyant sur la dimension factuelle et rationnelle (Okazaki, Mueller et Taylor, 2010). Les publicitaires doivent-ils rassurer le consommateur en donnant des preuves, des faits, des chiffres qui démontrent la qualité du produit ou service promu? Doivent-ils, au contraire, faire rêver les consommateurs, les sortir de leur consommation ordinaire en mettant au second plan le produit promu?

Cette problématique agite les académiques et les praticiens depuis plusieurs décennies déjà : «Comme la presse professionnelle capturait la tension, il s'agissait souvent d'un conflit entre " hard sell » et «soft sell », ou « école revendiquée » et "école de l'image 》. Indépendamment des termes spécifiques, la publicité rigoureuse, didactique pour Anacin ou Rolaids de Reeves représentait l'approche de hard sell, tandis que les campagnes Hathaway ou Schweppes plus sophistiquées et nuancées d'Ogilvy représentaient l'approche de soft sell. La valeur relative de ces deux styles était au centre du débat sur la créativité publicitaire et son efficacité globale à l'échelle de l'industrie dans les années 1950 et 1960. (Haygood, 2016, p. 170).

Compte tenu de sa capacité à faire évoluer les attitudes et à orienter les comportements des consommateurs, l'efficacité de la communication persuasive est l'une des thématiques les plus largement abordées par les chercheurs en marketing. Les recherches antérieures ont démontré l'efficacité des publicités émotionnelles (Geuens et DePelmarcker, 2002) et rationnelles (Yoo et McInnis, 2005 ; Hornik, Ofir et Rachamin, 2016) mais elles ont été menées généralement dans un contexte anglo-saxon (Alden, Steenkamp et Batra, 1999 ; Okazaki et al., 2010) ou une problématique de développement international (Mendel, 1994) alors même que ces recherches soulignent que l'efficacité publicitaire est fortement influencée par le contexte situationnel et culturel.

De manière surprenante au regard de leur poids grandissant dans l'économie mondiale et du dynamisme de ses marchés (Karimova, 2012), les pays arabo-musulmans, et plus 
particulièrement le Maghreb, ont rarement été étudiés. Le lancement par Emerald Group Publishing du Journal of Marketing islamique en 2010 ainsi que l'émergence de la publicité dans ces pays montrent un réel besoin dans ce domaine. Aussi, la culture arabo-musulmane se distingue d'une part, par les spécificités de la religion musulmane mais également par une identité culturelle (Perry et Motley, 2010). En effet, les spécificités de la religion musulmane doivent être prises en compte et impliquent manifestement des adaptations indispensables dans la commercialisation des entreprises qui souhaitent développer leurs activités dans ces pays (Pras et Vaudour-Lagrâce, 2007). Dans ce contexte, le monde arabe représente une entité spécifique en raison d'une très longue histoire culturelle et un langage commun qui conduisent notamment à la question de la standardisation ou l'adaptation des publicités, en raison de l'importance des liens entre la culture et les communications réalisées. Découle ainsi de ce constat la question principale : quelle est l'approche publicitaire utilisée dans les pays arabomusulmans? Plus exactement quel contenu publicitaire privilégié, plutôt une publicité qui joue sur les sens de la cible ou des contenus publicitaires plus informatifs ou rationnels?

Tel est l'objectif de cet article. Il vise à étudier les pratiques publicitaires dans le contexte arabomusulman, et plus particulièrement, à identifier si une approche publicitaire (soft-sell versus hard-sell) est privilégiée par les annonceurs. Cette étude répond à des préoccupations managériales importantes. Comprendre de manière approfondie les pratiques publicitaires des annonceurs dans un contexte arabo-musulman peut permettre de déterminer l'approche publicitaire à adopter dans le cas d'un lancement d'un nouveau produit à l'échelle nationale voire internationale.

L'article est structuré de la manière suivante. Dans une première partie, le cadre conceptuel est proposé et croise les notions d'approches publicitaires et de contexte culturel. La démarche méthodologique adoptée est présentée et justifiée dans la seconde partie. Les résultats sont alors détaillés. Finalement, la conclusion discute les limites de cette étude exploratoire, dresse les implications managériales et propose les voies futures de recherche. 


\section{Cadre conceptuel de la recherche}

La présente recherche croise le cadre théorique des deux approches publicitaires (hard-sell versus soft-sell) ainsi que le contexte culturel dans lequel se développe le discours publicitaire. La confrontation de ces deux champs de littérature permet de discuter des propositions de recherche qui guideront l'étude exploratoire.

\subsection{Contours et caractéristiques des deux approches publicitaires}

Ces approches publicitaires ont une longue histoire. D'après Beard (2004), la première tentative de définition remonte à 1911 lorsque les annonceurs automobiles américains ont débattu sur les mérites d'annonces fondées sur «the reason why » comparativement à des annonces mettant en valeur l'atmosphère (publicité impressionniste) dans le principal journal commercial de l'industrie « Printer's Ink ».

L'approche publicitaire hard-sell se réfère à une approche plus directe avec un accent sur un message rationnel centré sur le produit et des bénéfices utilitaires alors que l'approche publicitaire soft-sell est fondée sur des éléments plus subtiles et indirects s'appuyant davantage sur les émotions (Alden et al., 1999).

S'appuyant sur les travaux de Mueller (1987), Okazaki et al. (2010) proposent les définitions suivantes et délimitent ces approches par des critères de forme et de fond.

- L'approche publicitaire hard-sell est «celle dans laquelle l'objectif est d'activer la pensée rationnelle du récepteur du message. Ces messages ont tendance à être directs, mettant l'accent sur une orientation des ventes, et souvent en précisant le nom de la marque et les caractéristiques du produit. Il est souvent mentionné explicitement des informations factuelles, telles que des comparaisons avec des produits concurrents ou des traits distinctifs spécifiques du produit qui lui donnent un avantage dans les performances ou dans une autre dimension pertinente pour les consommateurs $»\left(\right.$ p. 7) ${ }^{1}$. L'utilisation des faits, la favorisation de la pensée et l'explicite constituent les trois piliers distinctifs de l'approche publicitaire hard-sell.

- L'approche publicitaire soft-sell est «celle dans laquelle les émotions humaines sont sollicitées pour induire une réaction affective du consommateur. Ces messages ont tendance à être subtils et indirects, une image ou une ambiance peuvent transporter à

\footnotetext{
1 "A hard-sell appeal is one in which the objective is to induce rational thinking on the part of the receiver. These appeals tend to be direct, emphasizing a sales orientation, and often specifying the brand name and product recommendations. There is often explicit mention of factual information, such as comparisons with competing products or specific distinguishing features of the product that give it an advantage in performance or some other dimension relevant to consumers."
} 
travers une belle scène ou le développement d'une histoire émouvante, ou via un autre mécanisme indirect» (p. 7) ${ }^{2}$. L'image, l'implicite et les sentiments constituent les trois piliers distinctifs de l'approche publicitaire soft-sell.

Encadré 1 : Illustration d'une affiche publicitaire de Burger King selon les deux approches
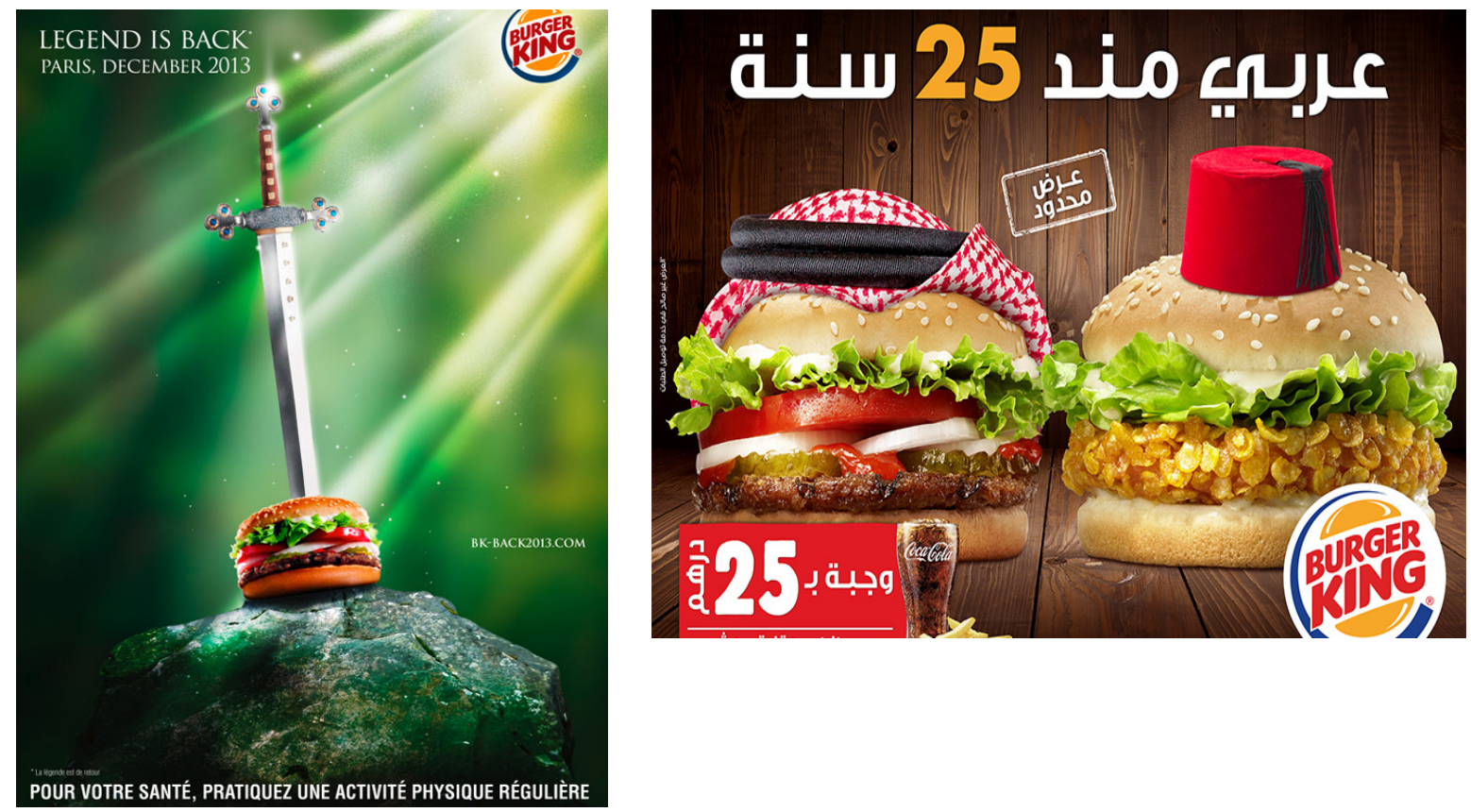

Burger King focalise son discours ${ }^{3}$ sur les qualités gustatives et le prix de son hamburger (photographie de droite) : l'approche publicitaire peut être qualifiée de hard-sell. En revanche, la photographie de gauche fait référence à Escalibur, la célèbre épée du Roi Arthur et au monde de la chevalerie moyenâgeuse pour annoncer son retour en France (« la légende est de retour »). Dans cette affichage, Burger King adopte une approche publicitaire soft-sell.

Ces approches publicitaires ont été largement discutées dans la littérature marketing, y compris de manière récente (Beard, 2004 ; Arora and Brown, 2012 ; Selvakumar et Mathew, 2015 ; Butt et al., 2017).

\subsection{Approches publicitaires et contexte culturel}

Le débat sur la standardisation de la publicité est relativement ancien : d'un côté, Elinder (1965) et Fatt (1967) alléguaient que l'évolution des sociétés entraîne inexorablement une convergence de ces dernières et une élimination des barrières culturelles permettant d'envisager et de justifier

\footnotetext{
2 "A soft-sell appeal is one in which human emotions are emphasized to induce an affective (feeling) reaction from the viewer. These appeals tend to be subtle and indirect, and an image or atmosphere may be conveyed throught a beautiful scene or the development of an emotional story, or via some other indirect mechanism."

${ }^{3}$ La traduction dans l'ordre du texte inscrit en arabe est : « Arabe depuis 25 ans »; « offre limitée »; " menu à 25 dirhams ».
} 
la standardisation de la communication ; et, ce d'autant plus, au regard des économies d'échelle inhérentes à la standardisation de la publicité (Levitt, 1983). D’un autre côté, Roostals (1963) note qu'en raison des spécificités culturelles des sociétés, la standardisation est difficile. Quant à Buzzel (1968) ou plus récemment Quelch et Hoff (1986), ces auteurs remarquent que les différences entre les marchés locaux encourageraient la vision d'une adaptation de la communication.

Alden et al. (1999) présument que l'approche publicitaire soft-sell conduit à une compréhension homogène et une bonne perception dans les différents marchés cibles, tandis que l'approche publicitaire hard-sell n'est pas percue similairement dans différents contextes culturels. Par ailleurs, Baalbaki et Malhotra (1993) avancent l'argument d'une standardisation de la communication face à des segments internationaux homogènes. Néanmoins, se pose la question de la définition de segments internationaux homogènes? Plus récemment et de manière contradictoire par rapport aux travaux antérieurs, la recherche de Butt et al. (2017) montre que l'approche publicitaire soft-sell n'est pas perçue de manière homogène par les consommateurs de trois pays (Pakistan, Etats-Unis et Japon) et les auteurs appellent à un approfondissement des recherches.

Les concepts de cultures à bas et haut contexte, introduits par les travaux de Linton (1945) et Hall (1976) pourraient expliquer les différences d'évaluation des approches publicitaires. Hall (1976) propose, en effet, d'apprécier toutes les cultures du monde selon un continuum entre bas et haut contexte. Pour ce faire, il distingue d'une part, le niveau visible et observable (qui correspond aux coutumes, rites et pratiques culturelles) ainsi que d'autre part, le niveau invisible et tacite (qui repose essentiellement sur les perceptions de l'espace et du temps et le rapport à l'autorité). De manière schématique, dans une culture de haut contexte, la communication s'inscrit essentiellement dans le contexte de communication lui-même, la communication non-verbale prime alors sur le message. Ainsi, les émotions et les ressentis ont une place primordiale. Dans une culture de bas contexte, la communication repose sur des informations détaillées et explicites ainsi que sur des raisonnements verbaux. Dans cette perspective, il est à noter que les cultures à haut contexte apprécient les messages lents et indirects (ce qui caractérise l'approche soft-sell), tandis que les cultures à bas contexte aiment plus les messages rapides et directs (ce qui caractérise l'approche hard-sell).

Reprenant cette dichotomie, Singh et Matsuo (2004) voient l'approche hard-sell comme une dimension de la culture à bas contexte, ils la décrivent comme des « réductions, promotions, coupons et l'accent mis sur les avantages du produit en utilisant la comparaison explicite » (p. 866). A leurs yeux, l'approche soft-sell est une dimension de la culture à haut contexte recourant 
à l'" utilisation des impressions affectives et subjectives, des aspects intangibles d'un produit ou d'un service et plus de divertissement pour promouvoir le produit » (p. 866).

\subsection{Vers une formulation des propositions de recherche}

La confrontation des concepts théoriques précédents permet de formuler deux propositions de recherche qui apparaissent contradictoires.

En s'appuyant sur la théorie proposée par Hall (1976) sur les cultures à bas et à haut contexte, il est à noter que « la communication y est informelle, subjective, souvent floue et non verbale comme en Asie, en Afrique ou au Moyen-Orient » (Meier, 2019, p. 48). A ce titre, les pays arabes peuvent être qualifiés de culture à haut contexte. En reprenant la proposition de Singh et Matsuo (2004), l'approche publicitaire retenue dans les pays arabo-musulmans devrait être majoritairement soft-sell.

Par ailleurs, l'islam a défini «des règles relatives à la conduite des affaires, à la liberté de l'économie de marché et à la résolution de problèmes dans les échanges de marchandises » (Pras et Vaudour-Lagrâce, 2007, p. 203) et dans les pays musulmans, la place de la religion reste forte. Dans ce contexte, il est inenvisageable qu'une entreprise puisse réaliser et diffuser une publicité sans prendre en compte le contexte et les valeurs locales, même si la cible semble adopter un comportement homogène dans tous les pays du monde. A titre d'illustration, en Malaisie, la publicité d'Unilever a été largement critiquée parce qu'elle montrait une femme séduire un homme grâce à son teint attrayant obtenu grâce à l'utilisation de la crème hydratante promue (Haque, Ahmed et Jahan, 2010). Ainsi, la prise en compte des valeurs de la charia islamique ainsi que la mise en avant de la qualité et de l'intégrité du produit est, selon certains chercheurs, inévitable dans la conception d'annonces dans des pays musulmans (Ahasanul et al, 2010 ; Razzouk et Al-Khatib, 1993 ; Luqmani et al, 1989). En s'appuyant sur ces différents exemples, il serait préférable, dans les pays musulmans, de proposer une annonce respectant les codes et usages religieux et qui présente les aspects factuels de l'offre. La communication correspond « en une information complète, précise et vraie, permettant une prise de décision fondée sur la nature réelle du produit » (Pras et Vaudour-Lagrâce, 2007, p. 204). De même, Rice et Al-Mossawi (2002) indiquent que les publicités dans le Golfe Persique utilisent moins l'exagération que dans les pays occidentaux.

Dans cette perspective, l'approche publicitaire retenue dans les pays arabo-musulmans devrait être majoritairement hard-sell. 
Pour résumer, sont ainsi identifiés des arguments pour défendre l'emploi d'approches publicitaires hard-sell, démontrant les avantages concrets du produit dans le monde arabomusulman, et d'autre part, des arguments opposés encourageants plutôt l'utilisation de l'approche publicitaire soft-sell. Cette contradiction amène à rechercher des éléments de compréhension supplémentaires en se focalisant sur l'étude des pratiques réelles des annonceurs.

\section{Méthodologie de la recherche}

Pour analyser et identifier l'approche publicitaire employée dans un contexte arabo-musulman, une étude qualitative exploratoire a été menée. Pour ce faire, des publicités télévisuelles ont été collectées au Maroc et en Arabie Saoudite et ont été analysées.

\subsection{Justifications du terrain d'étude}

Le Maroc et l'Arabie Saoudite ayant respectivement 32,2 et 28,2 millions d'habitants (Mahajan, 2014) ont été retenus pour la présente étude. Trois raisons essentielles justifient le choix de ces deux pays : leur position géographique, la pratique religieuse et les influences linguistiques.

En effet, le Maroc se situe au sud de l'Europe et de par son positionnement géographique et son histoire, ce pays entretient des liens étroits avec l'Europe, et plus particulièrement la France. L'Arabie Saoudite est géographiquement et historiquement plus éloignée de l'Europe, elle se situe au Moyen Orient et a reçu une influence plutôt anglophone.

Comme le souligne Bourqia (2010), le Maroc s'ancre dans l'islam qui « a façonné la vision du monde, le mode de vie et les traditions» (p. 110) mais, dans le même temps, «la société marocaine a subi de grandes transformations depuis l'Indépendance. Il s'ensuit que la sphère des valeurs a connu, par voie de conséquence, un changement » (p. 105). Depuis les années 60, le consommateur marocain a connu « une évolution remarquable surtout avec l'accélération de son niveau de revenu, les changements de ses modes de consommation, le développement et l'ouverture du marché national » (Bennani et Baakil, 2012, p. 39). Sans renier un profond attachement à des valeurs traditionnelles telles que la famille ou la religion, le consommateur marocain, et plus largement, le consommateur arabe actuel introduit des valeurs de modernité (Mahajan, 2014). Ainsi, les enseignements de l'islam sont appliqués de manière modérée au Maroc, contrairement à l'Arabie Saoudite où les pratiques apparaissent beaucoup plus conservatrices. 
Au niveau de la communication commerciale, les annonceurs doivent tenir compte de plusieurs contraintes comme l'indique le directeur général d'Independant Productions (Liban). «Les spots produits pour le marché saoudien doivent respecter les règles de la censure en vigueur en Arabie Saoudite : pas de scènes osées, se plier aux us et coutumes locales, etc. Il est ainsi interdit de manger ou de saluer avec la main gauche, traditionnellement réservée aux ablutions. Les femmes sont systématiquement voilées et vêtues de vêtements colorés lorsqu'elles sont à l'intérieur du foyer, mais elles sont obligatoirement habillées en noir à l'extérieur. Lorsqu'il s'agit d'une publicité pour un shampooing, par exemple, on filmera la femme de dos. On verra ses cheveux, mais pas son visage. $»^{4}$

Dans le respect de ce cadre, les publicitaires peuvent faire preuve de créativité. Au Maroc, le secteur publicitaire « se distingue ces dernières années par des manifestations de plus en plus rayonnantes » (Boutahri, 2018, p. 14) et la question de l'intégration des valeurs locales et globales apparaît tout à fait pertinente. En ce qui concerne la langue des publicités, les professionnels au Maroc ont recours à un mélange entre l'arabe classique, l'arabe dialectal, le français et l'anglais pour attirer le public le plus vaste possible (Erraoui, 2015).

\subsection{La sélection du corpus de publicités}

Les publicités institutionnelles ou centrées sur la marque employeur n'ont pas été considérées dans ce corpus puisque la cible n'est pas le consommateur final et que l'objectif n'est pas d'améliorer la notoriété et, in fine, l'achat.

Afin de renforcer la validité externe de l'étude, deux catégories de produits de consommation ont été sélectionnées : d'une part, les produits alimentaires et d'autre part, les produits d'hygiène et d'entretien. Les produits de luxe ou qui répondent à une consommation ostentatoire (automobile, vêtement, électroménager, etc.) ont été écartés car ils sont des symboles des pays industrialisés et ne sont consommés seulement que par une élite dans les pays étudiés. De plus, l'approche publicitaire internationale des produits de luxe s'appuie généralement sur une stratégie de standardisation. Il est à noter que le consommateur marocain «se voit attiré par les produits étrangers selon son pouvoir d'achat au détriment du produit fabriqué localement avec lequel il garde en esprit une mauvaise réputation (mauvaise qualité ou un mauvais rapport qualitélprix) » (Bennani et Baakil, 2012, p. 40).

Ont ainsi été retenus les produits alimentaires, d'hygiène et d'entretien qui s'ancrent dans les pratiques de consommation quotidiennes et concernent l'ensemble des consommateurs : les

\footnotetext{
${ }^{4}$ https://observers.france24.com/fr/20090717-publicite-version-saoudienne-censure
} 
produits alimentaires pour les marques locales car ce sont des produits de première nécessité que le Maroc et l'Arabie Saoudite produisent abondamment pour ne pas dépendre des importations et les produits d'hygiène et d'entretien car ce sont des produits pour lesquels les exportations se font plus facilement. 18 publicités ont été étudiées et émanent d'entreprises locales (10 publicités) et d'entreprises internationales ( 8 publicités). Dans la constitution de ce corpus, un strict équilibre entre Maroc et Arabie Saoudite a été retenu au sein des deux catégories étudiées. Bien qu'il s'agisse d'un échantillon de convenance, ces marques bénéficient d'une large notoriété, assurée par une couverture médiatique importante. A titre d'illustration, la marque Procter et Gamble investit entre 8 et $10 \%$ de son chiffre d'affaires en communication, assurant ainsi une place de leader à ses marques (Pampers, Tide, Pantène) dans leur catégorie ${ }^{5}$.

Le tableau 1 récapitule l'ensemble des publicités étudiées. Seules ont été observées et étudiées les publicités télévisuelles afin de constituer un corpus comparable. En effet, les impératifs de créativité et de conception liés au format de publicité (affichage, publicité magazine, radio, cinéma ou télévision) pourraient expliquer les variations observées dans l'approche publicitaire retenue. La télévision apparaît comme un média largement populaire et offre le plus grand potentiel de créativité. Il est, par conséquent, plus susceptible de révéler des différences.

L'ensemble des publicités sélectionnées a été ensuite enregistré en vue du codage des données.

Tableau 1 : Présentation des publicités étudiées

\begin{tabular}{|c|c|c|}
\hline \multicolumn{3}{|c|}{ MARQUES LOCALES } \\
\hline \multirow[b]{2}{*}{$\begin{array}{l}\text { Produits } \\
\text { alimentaires }\end{array}$} & Publicité au Maroc & Publicité en Arabie Saoudite \\
\hline & $\begin{array}{l}\text { - Thé (Sultan) } \\
\text { - Flan (Frosty) } \\
\text { - Margarine (Lilia) } \\
\text { - Yaourt à boire (Jamila) } \\
\text { - Huile d'olive (Alhora) }\end{array}$ & $\begin{array}{l}\text { - Poulet (Attazaj) } \\
\text { - Milk Shake (Almarai) } \\
\text { - Petit lait (Almarai) } \\
\text { - Huile de table (Savola) } \\
\text { - Jus de pommes (Almarai) }\end{array}$ \\
\hline \multicolumn{3}{|c|}{ MARQUES INTERNATIONALES } \\
\hline \multirow[b]{2}{*}{$\begin{array}{l}\text { Produits d'entretien } \\
\text { et d'hygiène }\end{array}$} & Publicité au Maroc & Publicité en Arabie Saoudite \\
\hline & $\begin{array}{l}\text { - Couches (Pampers) } \\
\text { - Lessive (Tide) } \\
\text { - Crème épilatoire (Vett) } \\
\text { - Shampooing (Pantène) }\end{array}$ & $\begin{array}{l}\text { - Couches (Pampers) } \\
\text { - Lessive (Tide) } \\
\text { - Crème épilatoire (Vett) } \\
\text { - Shampooing (Pantène) }\end{array}$ \\
\hline
\end{tabular}

\subsection{Processus de codage}

Après un «visionnage » flottant de l'ensemble du corpus, celui-ci a été codé à l'aide d'une grille d'observation qui est présentée en annexe 1. Celle-ci a été établie à partir du cadre

\footnotetext{
${ }^{5} \mathrm{https}: / /$ www.lavieeco.com/economie/pg-700-000-paquets-de-tide-et-300-000-dariel-vendus-chaque-jour$12351 /$
} 
conceptuel d'Okazaki et al. (2010). Comme présenté dans la revue de la littérature, ces auteurs délimitent les approches soft-sell et hard-sell à l'aide de trois dimensions qui sont respectivement d'une part, l'image, le sentiment, l'implicite; et d'autre part, l'explicite, la pensée, les informations factuelles.

Pour quantifier le degré de présence de ces dimensions, l'approche suggérée par Lin (2001) a été utilisée avec quatre échelons traduits par les valeurs numériques de 0 à $3: 0$ indiquant l'inexistence de cet élément à 3 qui indique une forte présence. Le codage a été réalisé par deux juges spécialistes en marketing et en communication persuasive.

Après une explicitation des différents critères qui délimitent les deux approches, les deux juges, ont codé séparément le corpus. Le rapprochement de leur codage souligne un fort accord spontané entre eux (supérieur à $95 \%$ ). Les désaccords ne portent pas sur la présence ou l'absence du critère mais bien sur l'appréciation de l'intensité. L'annexe 2 restitue la synthèse du codage des deux juges.

Tableau 2 : Présentation de la grille d'observation

\begin{tabular}{|l|l|l|l|}
\hline \multicolumn{2}{|c|}{ Approche publicitaire soft-sell } & \multicolumn{2}{c|}{ Approche publicitaire hard-sell } \\
\hline L'image & $\begin{array}{l}\text { Fondé sur l'imaginaire } \\
\text { Raconte une histoire }\end{array}$ & L'explicite & $\begin{array}{l}\text { Informe sur le prix } \\
\text { Invite explicitement à } \\
\text { l'achat } \\
\text { Présente une famille ou un } \\
\text { groupe } \\
\text { Utilise une vedette connue }\end{array}$ \\
\hline $\begin{array}{l}\text { Le } \\
\text { sentiment }\end{array}$ & $\begin{array}{l}\text { Fondé sur le divertissement } \\
\text { Fait passer un message } \\
\text { émouvant }\end{array}$ & La pensée & $\begin{array}{l}\text { Fait appel à la raison de } \\
\text { l'individu } \\
\text { Compare les produits }\end{array}$ \\
\hline L'implicite & $\begin{array}{l}\text { Utilise une scène humoristique } \\
\text { Utilise une vedette connue } \\
\text { Présente une famille ou un } \\
\text { groupe }\end{array}$ & $\begin{array}{l}\text { Les } \\
\text { informations } \\
\text { factuelles }\end{array}$ & $\begin{array}{l}\text { Montre l'utilisation du } \\
\text { produit } \\
\text { Montre longuement le } \\
\text { produit } \\
\text { Met en avant les atouts du } \\
\text { produit }\end{array}$ \\
\hline
\end{tabular}

\section{Résultats}

Les résultats de cette recherche sont structurés en deux parties. Premièrement, les marques internationales semblent adopter une stratégie de standardisation de leur communication télévisuelle et une approche publicitaire hard-sell. Deuxièmement, les marques locales adoptent, certes, une approche publicitaire hard-sell mais peuvent également recourir à une approche publicitaire soft-sell. 
3.1. Une stratégie de standardisation et une approche hard-sell pour les marques internationales

Le premier résultat concerne les stratégies publicitaires des marques internationales. L'observation des publicités relatives aux marques internationales diffusées au Maroc et en Arabie Saoudite fait ressortir très peu de différences entre elles et semble ainsi indiquer que les marques internationales retiennent une stratégie de standardisation pour leur communication.

De plus, les publicités de l'échantillon étudié mettent en évidence les avantages du produit (prix, modèles, qualités intrinsèques, etc.) et se concentrent peu sur les éléments périphériques (image de marque, bénéfices ostentatoires, etc.). La description des caractéristiques des produits et leur utilisation sont à chaque fois clairement mentionnées.

A titre d'illustration, les deux publicités promouvant les couches Pampers apparaissent très similaires entre le Maroc et l'Arabie Saoudite. Elles s'attachent à décrire l'usage et les bénéfices du produit de manière très factuelle. Elles adoptent le même scénario et le même message en mettant en scène un bébé et sa maman. De la même manière, les deux publicités relatives à la poudre à laver (Tide) se déroulent selon le même scénario : un enfant avec des vêtements sales dont la mère trouve dans la lessive Tide la solution idéale. Néanmoins, la publicité diffusée au Maroc a recours à une forme comparative indirecte (Dianoux, Herrmann et Zeitoun, 2013) contrairement à la publicité diffusée en Arabie Saoudite. Il est à noter également que les codes religieux et traditionnels sont respectés : les femmes portent des vêtements traditionnels et dans la publicité saoudienne, elle porte également un voile. Ceci est conforme à la stratégie publicitaire de Tide qui maîtrise ses aspects dans ses publicités : «Dans les années 1990, lorsque Procter \& Gamble a lancé Tide liquide sur les marchés arabes, l'entreprise décida d'utiliser toute une série de publicités montrant à quel point il était facile pour les femmes de se servir de ce produit pour laver leurs voiles. Elle prit soin de présenter la tâche comme une corvée quotidienne sans sous-entendu religieux, faisant ainsi clairement comprendre qu'elle percevait les rapports entre la culture et le business sur le marché arabe » (Mahajan, 2014, p. 3).

Les deux autres produits (Pantène et Veet) concernent le soin du corps dont l'islam préconise une représentation stricte. Pour le shampooing Pantène, la similarité entre les deux publicités est encore plus forte que dans les cas précédents puisque la marque a recours à la même star, qui est une actrice turque dont plusieurs films à grand succès ont été diffusés sur les chaînes arabes, pour vanter les mérites du produit ainsi qu'à la même langue (dialecte libanais). La publicité montre, ici, le résultat de l'utilisation du produit -des cheveux lisses et brillants- avec pour objectif de convaincre le consommateur que l'on ne peut changer de Pantène que pour un 
autre Pantène. L'argumentation se résume ainsi : «Est-ce possible qu'il existe mieux que Pantène? Mieux que Pantène, c'est le nouveau Pantène ». Ceci peut se comprendre comme une comparaison entre deux modèles de la même marque, une argumentation logique qui s'inscrit dans une approche publicitaire hard-sell.

Enfin, la publicité de la crème épilatoire Veet peut être analysée de la même manière. Est cependant illustré le rapport à l'islam. Si la publicité marocaine montre l'efficacité du produit en dévoilant une partie du corps de la femme dénudée, la publicité saoudienne souligne l'efficacité du produit à l'aide de la repousse des poils.

Ces quatre comparaisons de publicités internationales diffusées au Maroc et à l'Arabie Saoudite montrent que les annonceurs ont tous adopté une approche publicitaire hard-sell. Ces observations conduisent donc à conclure que la pratique des annonceurs a tendance à correspondre aux recommandations formulées par Luqmani et al. (1989) et aux résultats de l'étude de Butt et al. (2017). Celle-ci conclut que dans un marché en développement, l'approche publicitaire hard-sell est perçue comme plus crédible et crée une intention légèrement plus élevée chez les consommateurs d'acheter le produit.

Ce résultat irait à l'encontre des préconisations qui pourraient être déduites de l'approche de Hall (1976). 


\begin{tabular}{|c|c|c|}
\hline Marque & Maroc & Arabie Saoudite \\
\hline Pampers & $\begin{array}{l}\text { Les caractéristiques de la couche (forme, fermeture, modèle) et } \\
\text { les formats proposés sont tout d'abord montrés. Une voix } \\
\text { féminine ajoute : «la peau de votre bébé est sensible et } \\
\text { nécessite le plus grand soin. Bébé ne sera pas bien s'il est } \\
\text { humide ». } \\
\text { Pampers Premium présente } 5 \text { avantages significatifs présentés } \\
\text { sous forme d'étoiles (absorption rapide, absence d'humidité, } \\
\text { peau douce, crème protectrice en aloé-véra, etc.). } \\
\text { L'explication de ces avantages est détaillée. }\end{array}$ & $\begin{array}{l}\text { La publicité montre une mère avec son bébé qui porte une } \\
\text { couche-culotte. Le bébé semble manifestement bien dans sa } \\
\text { couche. } \\
\text { Le message explique que la sensibilité de la peau des bébés } \\
\text { nécessite l'utilisation de couches Pampers parce qu'elles sont } \\
\text { conçues pour respecter la peau des bébés. } \\
\text { Les informations sur le produit (la forme de la couche, les cinq } \\
\text { étoiles en or) défilent pendant la publicité. }\end{array}$ \\
\hline Tide & $\begin{array}{l}\text { Une mère se plaint de son enfant qui a sali son habit avec de la } \\
\text { terre. Elle demande à l'épicier trois paquets de son détergent } \\
\text { habituel mais le commerçant lui propose un seul paquet de } \\
\text { lessive « nouvelle formule». } \\
\text { On voit alors deux bols, le premier avec la lessive Tide et l'autre } \\
\text { avec une autre lessive. } \\
\text { Le résultat avec Tide apparaît spectaculaire. }\end{array}$ & $\begin{array}{l}\text { Le spot télévisuel montre un enfant qui s'amuse avec deux amis } \\
\text { qui ramassent un fruit sur un arbre. Il rentre chez lui avec des } \\
\text { vêtements très sales. Sa mère le voyant garde son calme parce } \\
\text { qu'elle a confiance en Tide. } \\
\text { A la fin de l'annonce, nous voyons l'enfant propre, un zoom est } \\
\text { fait sur le paquet de lessive. }\end{array}$ \\
\hline Veet & $\begin{array}{l}\text { L'annonce montre une jeune fille dont la robe se bloque dans } \\
\text { une porte d'ascenseur. Elle n'hésite pas à rendre sa robe plus } \\
\text { courte en enlevant la partie inférieure de la robe. Cet incident } \\
\text { ne semble pas la déranger dans la mesure où ses jambes sont } \\
\text { bien épilées. } \\
\text { Puis, une voix explique que le produit est beaucoup plus doux } \\
\text { que le rasoir et qu'il présente des bénéfices deux fois plus } \\
\text { importants qu'un rasoir. Il est également donné quelques détails } \\
\text { sur les quatre étapes de l'utilisation du produit. }\end{array}$ & $\begin{array}{l}\text { La publicité montre deux femmes assises sur une structure } \\
\text { flottante qui ressemble à un nénuphar. } \\
\text { Ensuite, la scène montre deux poils (on ne voit pas directement } \\
\text { la femme) qui sont éliminés avec Veet et un rasoir. Le premier } \\
\text { va pousser beaucoup plus lentement que celui enlevé avec le } \\
\text { rasoir. }\end{array}$ \\
\hline Pantène & La publicité utilise une actrice turque dont plusieurs films à gra & $\begin{array}{l}\text { pour vanter } \\
\text { our objectif } \\
\text { donnée par } \\
\text { ». }\end{array}$ \\
\hline
\end{tabular}

Tableau 3 : Résumé des scénarios des publicités des marques internationales 


\subsection{Une approche publicitaire mixte pour les marques locales}

En ce qui concerne les publicités relatives à des marques locales, elles sont présentées de manière détaillée dans le tableau 4 pour les produits marocains et dans le tableau 5 pour les produits saoudiens.

Les dix publicités étudiées sont axées sur la mise en avant des atouts du produit, essentiellement en rassurant sur la qualité du produit et en indiquant le prix. De manière plus sporadique, la comparaison directe ou indirecte est utilisée. Globalement, ces publicités pourraient être considérées comme relevant de l'approche publicitaire hard-sell.

Cependant, nous pouvons voir que deux annonces de produits saoudiens peuvent être considérées comme des approches publicitaires soft-sell (le jus de pomme et le milk-shake), ce qui nous amènera à être prudents dans notre conclusion.

Tableau 4 : Descriptions des publicités relatives à des marques locales au Maroc

\begin{tabular}{|c|}
\hline Thé Sultan \\
\hline $\begin{array}{l}\text { La publicité montre les étapes de préparation du thé. } \\
\text { Elle montre également des groupes folkloriques très connus pour rappeler l'authenticité du } \\
\text { thé marocain. L'accent est mis sur le logo de la marque qui prend une bonne place sur l'écran, } \\
\text { il est composé du nom de la marque, du symbole (couronne) et de la date de création (1936). }\end{array}$ \\
\hline Flan Forsty \\
\hline $\begin{array}{l}\text { La publicité montre une salle de classe où les fruits symbolisent les élèves et le professeur } \\
\text { (ananas, orange, noix de coco, etc.). } \\
\text { La mangue (professeur) pose une énigme à laquelle les élèves (fruits) doivent répondre : } \\
\text { "Quel est le dessert à base de fruits, fortifiant et nutritif pour seulement } 5 \text { dirhams ? " } \\
\text { Le professeur écrit la réponse sur le tableau : Forsty + un demi-litre de lait }+20 \mathrm{cl} \text { de sucre = } \\
5 \mathrm{dh} \text { pour } 6 \text { personnes. }\end{array}$ \\
\hline Yaourt à boire Jamila \\
\hline $\begin{array}{l}\text { Le message explique que le véritable « Raybi » est « Jamila ». } \\
\text { Dans cette publicité, le mot « véritable » est répété trois fois. } \\
\text { Pendant ce temps, l'accent est mis sur la composition du produit (le meilleur lait et l'arôme } \\
\text { de grenadine) en indiquant que ceci constitue le secret de la recette unique « Raybi Jamila. » }\end{array}$ \\
\hline Huile d'olive Alhora \\
\hline $\begin{array}{l}\text { Un présentateur bien connu de la télévision marocaine explique qu'il a fait une grande tournée } \\
\text { dans les régions pour découvrir les secrets de la fabrication de l'huile d'olive. } \\
\text { Ensuite, il fournit des informations (en utilisant des mots arabes qui riment) sur les conditions } \\
\text { d'obtention d'une bonne récolte (terres, quantités d'eau modérées, expertise des gens de } \\
\text { collecte) qui correspondent aux conditions de production de Alhora d'huile d'olive. }\end{array}$ \\
\hline Margarine Lilia \\
\hline $\begin{array}{l}\text { Une voix pour les individus qui font leurs achats annonce « Lilia est la première et seule } \\
\text { margarine végétale entièrement non hydrogénée au Maroc ». } \\
\text { Les consommateurs se demandent : « que signifie hydrogénée ? ». Les explications sont alors } \\
\text { données et ajoutent que le produit est riche en vitamines A, E, D. Le message montre des } \\
\text { individus convaincus et qui décident d'acheter seulement Lilia et remercient pour les conseils. }\end{array}$ \\
\hline
\end{tabular}


Tableau 5 : Description des publicités relatives à des marques locales en Arabie Saoudite

\begin{tabular}{|c|}
\hline Poulet Attazaj \\
\hline $\begin{array}{l}\text { La publicité montre la préparation de poulet grillé et le poulet servi dans un plat avec du pain } \\
\text { traditionnel. } \\
\text { Le message est centré sur l'explication des avantages du produit : poulet qui est nourri de } \\
\text { plantes, épicé avec des légumes frais, grillé sur le feu pour retirer toute la graisse et garder la } \\
\text { chair, à moins de } 198 \text { unités caloriques, délicieux et nutritif. } \\
\text { L'accroche est : «iln'y a pas plus frais qu'«Attazaj».» }\end{array}$ \\
\hline Almarai (petit lait) \\
\hline $\begin{array}{l}\text { Une famille (enfants, parents, grands-parents) est réunie autour d'une table à manger. } \\
\text { L'enfant sert un verre de lait à son grand-père. } \\
\text { Le message indique que vous pouvez faire plusieurs choix dans la vie, mais pour ceux qui } \\
\text { veulent offrir la qualité, il y a un seul choix : Almarai. } \\
\text { A la fin de la publicité, une voix annonce : « la qualité nous réunit ». }\end{array}$ \\
\hline Almarai (milkshake) \\
\hline $\begin{array}{l}\text { L'annonce montre des silhouettes éclairées d'un groupe de jeunes hommes qui dansent sur } \\
\text { un mélange de musique arabe et occidentale. } \\
\text { Un des danseurs secoue le produit. } \\
\text { La publicité est silencieuse et aucun message verbal n'est présent. } \\
\text { Le produit est indiqué au début, pendant et à la fin de la publicité. } \\
\text { L'annonce montre également le contenu (lait aromatisé avec des pépites de chocolat). } \\
\text { Deux accroches ont été utilisées : "faites bouger votre monde » et « une qualité qui mérite } \\
\text { la confiance». }\end{array}$ \\
\hline Huile de table Savola \\
\hline $\begin{array}{l}\text { Le narrateur explique le processus de la production de l'huile «Afia », accompagné par des } \\
\text { images de champs de maïs, et de l'extraction de l'huile naturelle du bon maïs. } \\
\text { «Pour une alimentation saine (une femme qui cuisine) et une famille en bonne santé (une } \\
\text { famille autour d'une table), utilisez «Afia »». } \\
\text { Elle est faite avec du bon maïs et sans cholestérol. }\end{array}$ \\
\hline Almarai (jus de pomme) \\
\hline $\begin{array}{l}\text { La publicité montre une femme qui cherche un sac à main dans un magasin chic. } \\
\text { Le vendeur lui soumet plusieurs propositions, mais elle n'accepte pas. Enfin, elle aime à } \\
\text { première vue un sac rouge comme la pomme et assez grand pour transporter le grand } \\
\text { emballage de son jus de pomme. } \\
\text { Nous voyons la dame verser le jus dans un grand verre et boire avec un grand désir. Nous } \\
\text { pouvons voir de bonnes pommes rouges sur l'emballage comme une preuve de la qualité du } \\
\text { produit. La revendication est: «nous ne pouvons pas faire sans ». }\end{array}$ \\
\hline
\end{tabular}

Cette pratique managériale qui s'oriente vers l'utilisation de l'approche publicitaire hard-sell pourrait être expliquée par les spécificités culturelles de l'arabo-musulman. En effet, lorsqu'on se réfère aux dimensions de Hofstede (2001) décrivant les cultures des pays, nous constatons que le besoin de sécurité est relativement développé dans la culture arabo-musulmane et donc le consommateur arabo-musulman régira probablement mieux à l'approche hard-sell du fait qu'elle le rassure avec les informations sur l'offre. 


\section{Conclusion}

En réponse à notre question de recherche qui était « quelle est l'approche publicitaire utilisée dans les pays arabo-musulmans? ", il semble que l'approche publicitaire dans les deux pays étudiés est relativement homogène et que les publicités sont centrées sur l'utilisation des éléments de l'approche hard-sell.

La connaissance de ces pratiques apparaît utile pour les professionnels de la publicité tant nationaux qu'internationaux. Elle leur permet d'identifier les tendances publicitaires dans le contexte arabo-musulman et les conduit à réfléchir sur l'efficacité des approches adoptées tout en tenant compte des spécificités culturelles de ce marché.

Sur la base de ces observations, le postulat selon lequel les pays arabo-musulmans adoptent des communications fondées sur de l'implicite (selon la théorie interculturelle développée par Hall) ne semble pas être supporté sur la base de nos observations. Toutefois, la présence de deux publicités qui mettent en œuvre une approche publicitaire soft-sell dans l'échantillon d'Arabie Saoudite nous amène à être très prudents dans notre conclusion et nous conduit à considérer les limites de cette recherche. La première limite concerne l'échantillon retenu tant dans sa taille que dans ses caractéristiques. En effet, l'échantillon reste modeste (18 publicités télévisuelles analysées), concerne deux catégories de produit, s'attache à deux pays (Maroc et Arabie Saoudite) et concerne un seul support de communication (la télévision). Pour apporter une compréhension approfondie du processus de décision des annonceurs quant à l'approche publicitaire dans le monde arabo-musulman, ce travail mérite d'être élargi en considérant d'autres catégories de produits comprenant notamment des services, des produits durables et des produits industriels, d'autres pays et d'autres supports de communication (notamment, l'affichage ou la publicité online) pour une plus grande possibilité de généralisation. Ceci serait conforme à la recommandation de Cardon (2008, p. 423) qui souligne que « les chercheurs qui citent ou interprètent la communication interculturelle en termes de contexte doivent le faire avec prudence ». L'auteur recommande ainsi d'élaborer des mesures ou instruments pour mesurer le contexte, d'inclure plus de pays et de cultures dans les études, de développer les catégories, de se concentrer sur les circonstances dans lesquelles les différentes cultures utilisent à la fois les messages à haut contexte et à bas contexte. Au-delà du renforcement de la validité externe de l'étude, trois principales perspectives de recherche émergent. Tout d'abord, une étude qualitative à l'aide d'entretiens semi-directifs auprès des agences de communication et des annonceurs évoluant dans le monde arabo-musulman pourrait permettre de confronter les discours de ces acteurs-clés avec leurs pratiques managériales. Deuxièmement, il serait 
intéressant d'étudier l'impact des approches publicitaires soft-sell et hard-sell sur le comportement du consommateur arabo-musulman pour donner aux professionnels de la publicité dans ces pays un retour sur la capacité des deux approches à persuader leur cible. Cette étude quantitative permettrait de considérer simultanément les qualités de la marque (ancienneté et position sur le marché, nature du produit promu, répartition des achats entre les hypermarchés et sur les marchés traditionnels, concurrence, etc.) et les variables individuelles (respect de la religion et pratiques religieuses, implication, expertise, besoin de sécurité, etc). Enfin, étendre cette recherche aux pays occidentaux permettrait de révéler les différences saillantes en raison des spécificités culturelles de ces pays. Des investigations tant qualitatives que quantitatives apparaissent indispensables pour construire une compréhension approfondie de l'approche publicitaire considérant le contexte culturel. 


\section{Références bibliographiques}

Alden, D.L., Steenkamp J.B.E.M., et Batra R. (1999). Brand Positioning Through Advertising in Asia, North America, and Europe: The Role of Global Consumer Culture. Journal of Marketing, 63(1), 75-87.

Arora, A., \& Brown III, U. J. (2012). Hard-sell and soft-sell advertising appeals with a'Polysemic'difference: A purposeful advertising polysemy perspective. Advertising \& Society Review, 13(2).

Baalbaki, I.B. et Malhotra N. K. (1993). Marketing Management Bases for International Marketing Segmentation: An Alternative Look at the Standardization/Customization Debate. International Marketing Review, 10(1), 19-44.

Beard, Fred K. (2004)"Hard-Sell "Killers" and Soft-Sell "Poets" Modern Advertising's Enduring Message Strategy Debate." Journalism History 30.3 (2004): 141-149.

Bennani, B., et Baakil, D. 2012 Le comportement du consommateur au Maroc. Revue Economie \& Kapital, (1).

Bourqia, R. (2010). Valeurs et changement social au Maroc. Quaderns de la Mediterrània, 13, 105-115.

Boutahri, F. (2018) La communication publicitaire au Maroc : une naissance sous le masque du modernisme. Sciences, Langage et Communication, 2(1). P. 14

Butt, Irfan, Bhasker Mukerji, and Mahmud A. Shareef. "Relevance of soft-sell and hard-sell advertising appeals for global consumer cultural positioning." Journal of Customer Behaviour 16.3 (2017): 263-279.

Buzzel Robert D. (1968), Can You Standardize International Marketing? Harvard Business Review, 46, November/December, 103-113.

Cardon, P. W. (2008), A Critique of Hall's Contexting Model, Journal of Business and Technical Communication, 22, 4, 399-428.

Dianoux, C., Herrmann, J. L., et Zeitoun, H. (2013). Comparative advertising: citing or not the leading brand and its price. Journal of Consumer Marketing.

Elinder Erik (1965), How International Can European Advertising be? Journal of Marketing, 29, April, 7-11.

Erraoui, I. E. (2015). L'alternance codique dans le domaine de la publicité au Maroc. Langues, cultures et sociétés, 1(1), 60-78.

Fatt Arthur C. (1967), The Danger of Local International Advertising, Journal of Marketing, $31,1,60-62$. 
Geuens, M. et De Pelsmacker, P. (2002). The role of humor in the persuasion of individuals varying in need for cognition. Advances in Consumer Research, 50-56.

Hall Edward T. (1976), Beyond Culture, New-York, Anchor Books Editions.

Haque A., Ahmed K., et Syeada, I.J. (2010). Shariah observation:advertising practices of bank muamalat in malaysia. Journal of islamic marketing, 1, 1, 70-77.

Haygood, D. M. (2016). Hard Sell or Soft Sell? The Advertising Philosophies and Professional Relationship of Rosser Reeves and David Ogilvy. American Journalism, 33(2), 169-188.

Hofstede, G. (2001). Culture's consequences:comparing values, behaviors, institutions and organisations across nations. Australian Journal Of Management, 89-94.

Hornik, J., Ofir, C., et Rachamim, M. (2016). Quantitative evaluation of persuasive appeals using comparative meta-analysis. The communication Review, 192-222.

Karimova G. Z. (2012), Branding and advertising in an Islamic context: Representing the forbidden, Journal of Brand Strategy, 1, 4, 333-343.

Levitt T. (1983), The Globalizations of Markets, Harvard Business Review, 61, May/June, 92102.

Lin C. A. (2001). Cultural values reflected in chinese and american television advertising, Journal of Advertising, 30, 4, pp. 83-95.

Linton R. (1945), The cultural background of personality, D. Appleton-century Crofts.

Luqmani M., Quraeshi, Z. et Yavas, U. (1989), Advertising in Saudi Arabia: Content and Regulation, International Marketing Review, 31, 3, 35-39.

Mahajan V. (2014), Comprendre les consommateurs du monde arabe, Harvard Business Review, février-mars, 1-5.

Meier O., Management interculturel. Dunod, « Management Sup », 2019, 336 pages. ISBN : 9782100788897. DOI : 10.3917/dunod.meier.2019.01. URL : https://www.cairn.info/management-interculturel--9782100788897.htm

Mendel, D. (1994). Comparaison des cultures allemandes et françaises et implications marketing. Recherche et Applications en Marketing, 32-75.

Mueller, B. (1987). Reflections of culture-an analysis of Japanese and American advertising appeals. Journal of Advertising Research, 27(3), 51-59.

Okazaki, S., Mueller B., et Taylor C. R. (2010), Measuring soft-sell versus hard-sell advertising appeals, Journal of Advertising, 39, 2, 5-20.

Perry V. G., et Motley C. M. (2010), Dreams and Taboos: Home Loan Advertising in the United States and Saudi Arabia, Journal of International Consumer Marketing, 22, 2, 199-212. 
Pras, B. et Vaudour-Lagrâce, C. (2007). Marketing et islam. Revue française de gestion, (2), 195-223.

Quelch J. A. et Hoff E.J. (1986), Customizing Global Marketing, Harvard Business Review, 64, May/June, 59-68.

Razzouk, N. et Al-Khatib J. (1993), The Nature of Television Advertising in Saudi Arabia: Content Analysis and Marketing Implications, Journal of International Consumer Marketing, $6,2,65-90$

Rice, G. etAl-Mossawi M., "The Implications of Islam for Advertising Messages: The Middle Eastern Context", Journal of Euromarketing, vol. 11, n 3,2002, p. 71-97.

Roostals, I. (1963), Standardization of Advertising for Western Europe, Journal of Marketing, October, 15-20.

Selvakumar, J. Joshua, and Marina Mary Mathew. "The impact of hard sell and soft sell advertisements in altering viewers attitude and purchase intension towards brands." International Journal of Marketing and Technology 5.9 (2015): 214-228.

Singh, N. et Matsuo H. (2004), Measuring cultural adaptation on the Web: a content analytic study of U.S. and Japanese Web sites, Journal of Business Research, 57, 8, 864-872.

Yoo, C. et Maclnnis, D. (2005). The brand attitudes formation process of emotional and information advertising. Journal of business research, 1397-1406.

Zhou, Lianxi, Patrick Poon, and Haizhong Wang. "Consumers' reactions to global versus local advertising appeals: A test of culturally incongruent images in China." Journal of Business Research 68.3 (2015): 561-568. 


\section{Annexe 1: Grille d'observation}

Observation $n^{\circ}$ : Date et heure

Nom de l'observateur :

Nom de la marque :

Produit :

Type de produit : $\quad$ Alimentaire $\quad$ Entretien et hygiène

Durée de l'annonce :

Chaîne de diffusion :



Autres commentaires 


\begin{tabular}{|c|c|c|c|c|c|c|c|c|c|c|c|c|c|c|c|c|c|c|}
\hline & \multicolumn{10}{|c|}{ Marques locales } & \multicolumn{8}{|c|}{ Marques internationales } \\
\hline & \multicolumn{5}{|c|}{ Maroc } & \multicolumn{5}{|c|}{ Arabie Saoudite } & \multicolumn{4}{|c|}{ Maroc } & \multicolumn{4}{|c|}{ Arabie Saoudite } \\
\hline & 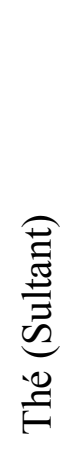 & 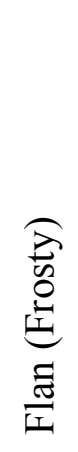 & 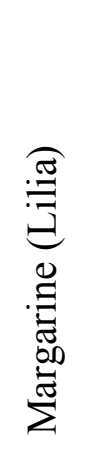 & 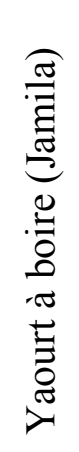 & 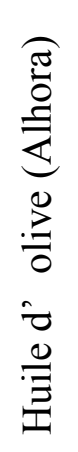 & 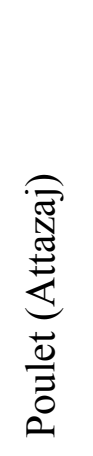 & 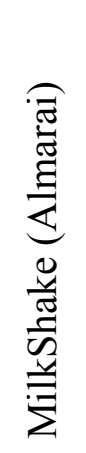 & 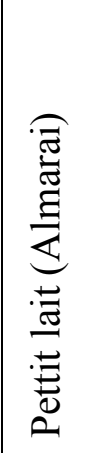 & 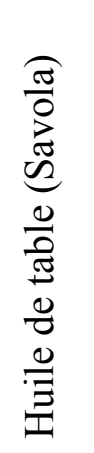 & 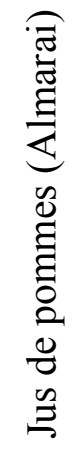 & 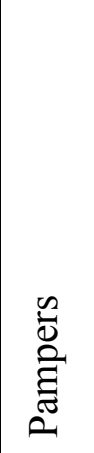 & $\underset{\mathscr{D}}{\mathbb{D}}$ & $\stackrel{\vec{D}}{>}$ & 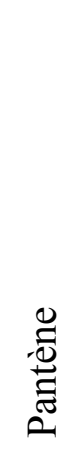 & 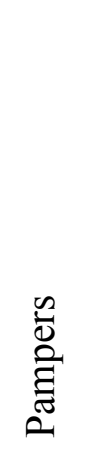 & $\stackrel{\mathscr{D}}{:}$ & $\stackrel{\mathbb{D}}{\nu}^{+}$ & 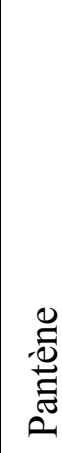 \\
\hline \multicolumn{19}{|l|}{ L'image } \\
\hline Fondé sur l'imaginaire & 0 & 3 & 0 & 0 & 0 & 0 & 0 & 0 & 0 & 0 & 0 & 0 & 0 & 0 & 0 & 0 & 0 & 0 \\
\hline \multicolumn{19}{|l|}{ Le sentiment } \\
\hline \multicolumn{19}{|l|}{ L'explicite } \\
\hline Utilise une vedette connue & 3 & 0 & 0 & 2 & 3 & 0 & 0 & 0 & 0 & 0 & 0 & 0 & 0 & 3 & 0 & 0 & 0 & 3 \\
\hline Présente une famille ou un groupe & $2 / 1$ & $2 / 3$ & $2 / 1$ & 3 & 0 & 0 & 3 & 3 & 1 & 0 & 3 & $2 / 3$ & 1 & 0 & 3 & 2 & 0 & 0 \\
\hline Informe sur le prix & 0 & 3 & 0 & 0 & 0 & 0 & 0 & 0 & 0 & 0 & 0 & 0 & 0 & 0 & 0 & 0 & 0 & 0 \\
\hline Invite explicitement à l'achat & 0 & 0 & 0 & 0 & 0 & 0 & 2 & 2 & 0 & 0 & 0 & 0 & 0 & 2 & 0 & 0 & 2 & 2 \\
\hline \multicolumn{19}{|l|}{ La pensée } \\
\hline Fait appel à la raison de l'individu & 0 & 1 & 3 & 1 & 1 & $1 / 2$ & $1 / 2$ & 1 & $1 / 2$ & 0 & 1 & 0 & 3 & 1 & 1 & 2 & 0 & 1 \\
\hline Compare les produits & 0 & 0 & 2 & 3 & 0 & 1 & 0 & 0 & 0 & 0 & 0 & 3 & 1 & 2 & 0 & 0 & 0 & 2 \\
\hline
\end{tabular}


Met en avant les atouts du produit

Montre longuement le produit

Montre l'utilisation du produit

\begin{tabular}{|l|l|l|l|l|l|l|l|l|l|l|l|l|l|l|l|l|l|}
\hline 0 & 3 & 3 & 2 & 3 & 3 & $1 / 2$ & 1 & 3 & 0 & 3 & 2 & 3 & 1 & 3 & 3 & 3 & 1 \\
\hline 3 & $2 / 3$ & 3 & 3 & 2 & 3 & 3 & 3 & 3 & 3 & 3 & 3 & 3 & 3 & 2 & 3 & $2 / 3$ & 3 \\
\hline 2 & 0 & 0 & 0 & 1 & 0 & 3 & 3 & 2 & 3 & 1 & 3 & 3 & 2 & 1 & $2 / 3$ & $2 / 3$ & 2 \\
\hline
\end{tabular}

